\title{
Cost-benefit comparison of liraglutide and sitagliptin in the treatment of type 2 diabetes in Thailand
}

This article was published in the following Dove Press journal:

ClinicoEconomics and Outcomes Research

\section{Chaicharn \\ Deerochanawong' \\ Natapong Kosachunhanun ${ }^{2}$ \\ Arvind V Gadekar ${ }^{3}$ \\ Pitthaporn Chotikanokrat ${ }^{3}$ \\ Unchalee Permsuwan ${ }^{4}$ \\ 'Rajavithi Hospital, College of Medicine, Rangsit University, Ministry of Public Health, Bangkok 10400, Thailand; ${ }^{2}$ Department of Medicine, Faculty of Medicine, Chiang Mai University, Chiang Mai 50200, Thailand; ${ }^{3}$ Novo Nordisk Pharma (Thailand) Ltd., Bangkok 10500, Thailand; ${ }^{4}$ Department of Pharmaceutical Care, Faculty of Pharmacy, Chiang Mai University, Chiang Mai 50200, Thailand}

Correspondence: Unchalee Permsuwan Department of Pharmaceutical Care, Faculty of Pharmacy, Chiang Mai University, Chiang Mai 50200, Thailand Email unchalee.permsuwan@gmail.com

\begin{abstract}
Aim: Liraglutide, a once-daily subcutaneous glucagon-like peptide-1 (GLP-1) agonist, is approved for treatment of hyperglycemia in patients with type 2 diabetes mellitus (T2DM). For patients with established cardiovascular diseases, liraglutide has also been shown to reduce major cardiovascular events. However, its cost is relatively higher than other oral antidiabetic drugs. This study aims to compare the costs and benefits of liraglutide vs sitagliptin, in treating T2DM in Thailand.
\end{abstract}

Methods: This study consists of two parts. In part 1, the cost of keeping T2DM under control per patient (HbA1c $<7.0 \%$ with no reported hypoglycemia and no body weight gain) with liraglutide (1.2 and $1.8 \mathrm{mg}$ daily) was compared with using sitagliptin (100 mg daily). Costs were based on Thai local data. Clinical outcomes were based on head-to-head randomized controlled trials. Part 2 estimated the cost-per-controlled patient, based on major cardiovascular outcomes (cardiovascular death, nonfatal myocardial infarction, nonfatal stroke). Economic benefit was calculated as the reduction in cardiovascular outcomes. Results: In Thailand, liraglutide (1.8 mg daily) costs 7.37-times more than sitagliptin $100 \mathrm{mg}$. The cost per patient achieving a composite clinical endpoint (HbAlc $<7.0 \%$, with no weight gain and no hypoglycemic events) in patients with T2DM receiving liraglutide $1.8 \mathrm{mg}$ is 2.80 -times higher than patients receiving sitagliptin $100 \mathrm{mg}$. When cardiovascular benefits (reduced composite endpoint of major cardiovascular events, ie, cardiovascular death, nonfatal myocardial infarction, and nonfatal stroke) were taken into account, it was found that liraglutide had lower cost than sitagliptin, resulting in estimated savings of 20,085 THB (620 USD) per patient per year.

Conclusion: The clinical benefits of liraglutide (HbA1c $<7.0 \%$, no hypoglycemia, no weight gain, reduced cardiovascular outcomes) partly offset its high price. Therefore, liraglutide should be considered as an appropriate treatment alternative to sitagliptin, particularly for T2DM patients with high cardiovascular risks.

Keywords: liraglutide, sitagliptin, diabetes, cardiovascular, Thailand

\section{Introduction}

The current pandemic of diabetes mellitus and the projected future growth of the prevalence of the disease constitute a global public health crisis. Furthermore, increasingly unhealthy lifestyles, attributed to poor diets, immobility, and the growing prevalence of obesity in developing countries, are contributing to its increased occurrence. ${ }^{1,2}$ For example, from 2009 to 2014, diabetes prevalence amongst $\geq 15$ year-olds increased from 6.9 to $8.9 \%$ (National Health Examination Survey (NHES) IV and NHES V, respectively ${ }^{3}$ ), whilst from 2012 to 2016, 
mortality rates of diabetes patients per 100,000 population almost doubled from 12.1 to $22.3,{ }^{4}$ putting the disease among the top ten leading causes of death in the country.

As the number of diabetes patients increases in Thailand, the costs of the disease and its complications have also increased. ${ }^{5}$ The average annual healthcare cost of a diabetic patient was USD 881.47 in 2008 or 948.57 presented in 2018 USD (which has increased to 956.16 in 2018 ), amounting to $21 \%$ of gross domestic product per capita. Complications from the disease increase costs substantially. Informal care, defined as the care provided by people from a care recipient's social network such as family, friends, neighbors, ${ }^{6}$ accounts for a greater percentage of overall costs than medical treatment $(28 \%$ vs $23 \%){ }^{7}$ indicating a substantial economic impact on patients' families. Therefore, effective interventions or initiatives are urgently needed to reduce both the incidence and the financial impact of the disease, as well as improve patients' quality-of-life.

Liraglutide is a glucagon-like peptide-1 (GLP-1) agonist. It is an effective antidiabetic drug that does not cause weight gain, a common side-effect associated with several other commonly used antidiabetic drugs. ${ }^{8}$ The drug provides greater glycemic control and weight reduction in T2DM patients than sitagliptin does. ${ }^{9,10}$ Moreover, it reduces the incidence of cardiovascular diseases and mortality of T2DM patients who have high cardiovascular risks, compared with placebo and other anti-diabetic drugs. ${ }^{11,12}$ Although liraglutide is a relatively new and premium drug, the benefits from its use could potentially outweigh its high price. Hence, the study presented here was aimed at comparing the costs and benefits of using liraglutide to treat T2DM patients in Thailand with using sitagliptin for the same purpose.

\section{Subjects, materials, and methods Scope of the study}

There were two parts to this study. Part 1 addressed the cost of achieving glycemic control in T2DM patients, whilst part 2 expanded to evaluate the impact that preventing adverse cardiovascular outcomes had on total cost of care.

Part 1 determined the average annual cost per patient of achieving the clinically relevant composite endpoint that indicates control of the disease: $\mathrm{HbAlc}<7.0 \%$, no reported hypoglycemia, and no body weight gain. ${ }^{9,10}$ The treatments compared were daily doses of liraglutide (1.2 $\mathrm{mg}$ or $1.8 \mathrm{mg}$ daily) or sitagliptin (100 mg daily).
The cost of control model was developed using Microsoft Excel. The time horizon was 52 weeks, based on the clinical outcomes from the clinical trial. Consequently, discounting was not considered in the study.

Part 2 extended the study to include the costs of cardiovascular outcomes in a cost-benefit comparison between liraglutide (1.8 $\mathrm{mg}$ daily) and sitagliptin (100 mg daily). The cost-per-controlled-patient was the medication cost of the significant adverse cardiovascular outcomes, including reduced composite endpoint events (cardiovascular death, non-fatal myocardial infarction (MI), and non-fatal stroke), death from cardiovascular causes, and myocardial infarction. In addition, the economic benefits arising from the prevention of such outcomes were also calculated.

\section{Treatment strategies}

During part 1 of the study, we compared liraglutide $1.8 \mathrm{mg}$ or $1.2 \mathrm{mg}$, administered by a once-daily subcutaneous injection, with sitagliptin $100 \mathrm{mg}$, taken orally also once daily; whilst, in part 2, the benefits of subcutaneous liraglutide (1.8 mg daily only) were compared with those of oral sitagliptin (100 mg daily).

\section{Treatment cohorts}

The cohort population used in part 1 of the study consisted of T2DM patients who had inadequate glycemic control ( $\mathrm{HbA} 1 \mathrm{c}>7.5 \%$ ), whilst those used for part 2 consisted of T2DM patients at high risk of cardiovascular diseases.

\section{Clinical data}

In part 1 of the study, the proportions of T2DM patients achieving the composite endpoint, which indicates control of the disease, were obtained from the 1860-LIRA-DPP4 trial (ClinicalTrial.gov Identifier: NCT00700817) published in 2010-2011., ${ }^{9,10}$ In brief, this head-to-head randomized controlled trial (RCT) conducted in Europe and North America enrolled T2DM patients, whose glycemic control (HbAlc 7.5-10.0\%) when treated with metformin ( $\geq 1500 \mathrm{mg}$ daily for $\geq 3$ months) was inadequate. Patients were randomly allocated to one of three treatment groups: subcutaneous liraglutide once daily, $1.2 \mathrm{mg}(\mathrm{n}=225)$ or $1.8 \mathrm{mg}(\mathrm{n}=221)$, or oral sitagliptin once daily, $100 \mathrm{mg}$ $(\mathrm{n}=219)$. The cohort population had a mean age \pm standard deviation (SD) of $55.3 \pm 9.2$ years, mean duration of diabetes of $6.0 \pm 4.5$ years, mean $\mathrm{HbAlc}$ of $8.4 \% \pm 0.8$, and mean body mass index (BMI) of $32.8 \pm 5.2 \mathrm{~kg} / \mathrm{m}^{2}$. 
The clinical data, used in part 2 of the study, were from two large previous studies: the LEADER trial $(9,430$ patients), published in 2016, and the TECOS trial (14,735 patients), published in 2015. The former examined the effects of liraglutide on cardiovascular outcomes of T2DM patients, ${ }^{11}$ whilst the latter administered the use of sitagliptin for the same objective. ${ }^{13}$ In each study, patients at high risk of cardiovascular disease from more than 30 countries were randomly assigned in a 1:1 ratio to be a placebo group or a drug-treatment group (liraglutide subcutaneous $1.8 \mathrm{mg}$ or sitagliptin oral $100 \mathrm{mg}$ ). The median follow-up time was 3.5 years for the LEADER trial and 3.0 years for the TECOS trial. Only statistically significant cardiovascular outcomes were included in analyses performed for the study, as presented below (Table 1).

\section{Costs}

Drug costs were calculated from daily doses and unit costs. Daily doses, used in cost calculations, were those in the LIRA-DPP4 trial, ${ }^{9,10}$ whilst the unit costs of the drugs were derived from the Royal Government Gazette of Thailand (February 23, 2018 ${ }^{14}$ ). The daily costs were $160.50 \mathrm{THB}$ (4.96 USD) and 240.75 THB (7.43 USD) for $1.2 \mathrm{mg}$ and $1.8 \mathrm{mg}$ liraglutide, respectively, and $32.68 \mathrm{THB}$ (1.01 USD) for sitagliptin (100 mg/tablet). Costs of cardiovascular events, such as fatal myocardial infarction, non-fatal myocardial infarction, and stroke, were obtained from the study conducted in Thailand. ${ }^{15,16}$ Each cost item was displayed in Table 2. All costs were presented at year 2018 values and converted at the rate of 32.39 THB per $\mathrm{USD}^{17}$ ).

\section{Analysis}

The cost of control of T2DM was defined as the cost of treatment divided by the specific outcome. In part 1, the cost of control was calculated from the cost of individual treatments divided by the percentage of patients achieving the composite endpoint. The ratio of composite endpoint between liraglutide and sitagliptin $>1$ indicated that liraglutide achieved the composite endpoint greater than sitagliptin did.

$$
\text { Ratio of treatment cost }=\frac{\text { cost of liraglutide treatment }}{\text { cost of sitagliptin treatment }}
$$

percentage achieving the

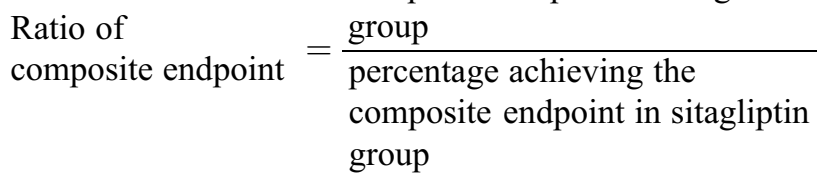

\begin{tabular}{|c|c|c|c|c|c|c|}
\hline \multicolumn{7}{|c|}{ Clinical Outcomes (Part I)* - percentage of patients achieving the composite endpoint ${ }^{a}$} \\
\hline Treatment & \multicolumn{3}{|l|}{ Value (\%) } & \multicolumn{3}{|l|}{ n } \\
\hline Liraglutide $1.2 \mathrm{mg}$ & \multicolumn{3}{|l|}{38.9} & \multicolumn{3}{|l|}{221} \\
\hline Liraglutide $1.8 \mathrm{mg}$ & \multicolumn{3}{|l|}{49.0} & \multicolumn{3}{|l|}{218} \\
\hline Sitagliptin $100 \mathrm{mg}$ & \multicolumn{3}{|l|}{18.6} & \multicolumn{3}{|l|}{219} \\
\hline \multicolumn{7}{|c|}{ Clinical Outcomes (Part II) - MACE (no. of events per 100 patients per year) } \\
\hline \multirow[t]{2}{*}{ Variable } & \multicolumn{3}{|c|}{ LEADER trial " } & \multicolumn{3}{|c|}{ TECOS trial ${ }^{13}$} \\
\hline & $\begin{array}{l}\text { Liraglutide } \\
1.8 \mathrm{mg}\end{array}$ & Placebo & P-value & $\begin{array}{l}\text { Sitagliptin } \\
100 \mathrm{mg}\end{array}$ & Placebo & $P$-value \\
\hline Incidence rate of composite endpoint ${ }^{b}$ & 3.4 & 3.9 & 0.01 & 3.58 & 3.62 & ns \\
\hline Reduction $^{c}$ & \multicolumn{3}{|l|}{0.50} & \multicolumn{3}{|l|}{0.04} \\
\hline Cardiovascular death & 1.2 & 1.6 & 0.007 & 1.72 & 1.67 & ns \\
\hline Reduction $^{c}$ & \multicolumn{3}{|l|}{0.40} & \multicolumn{3}{|l|}{$-0.05^{d}$} \\
\hline Incidence rate of myocardial infarction & 1.60 & 1.90 & 0.046 & 1.42 & 1.51 & ns \\
\hline Reduction ${ }^{c}$ & \multicolumn{3}{|l|}{0.30} & \multicolumn{3}{|l|}{0.09} \\
\hline
\end{tabular}

Table I Summary of clinical outcomes

Notes: *Data source: Pratley et al, $201 \mathrm{I} .{ }^{10}$ a Composite endpoint defined as achieving $\mathrm{HbAlc}<7.0 \%$, no hypoglycemia, and no weight gain. ${ }^{\mathrm{b}}$ Composite endpoint defined as death from cardiovascular causes, nonfatal myocardial infarction, nonfatal stroke. 'Difference number of events in placebo group-number of events in active group. ${ }^{\mathrm{d}}$ Patients in the sitagliptin group had a higher death rate from cardiovascular causes than those in the placebo group.

Abbreviations: mg, milligram; n, number; ns, not-significant; SD, standard deviation; THB, Thai baht. 
Table 2 Summary of cost inputs

\begin{tabular}{|c|c|c|}
\hline Cost items & Value THB (USD) & Data source \\
\hline \multicolumn{3}{|l|}{ Drug costs } \\
\hline Sitagliptin (100 mg per day) & $32.68(1.01)$ & Royal Gazette ${ }^{14}$ \\
\hline Liraglutide ( $1.2 \mathrm{mg}$ per day) & $160.50(4.96)$ & Royal Gazette ${ }^{14}$ \\
\hline Liraglutide ( $1.8 \mathrm{mg}$ per day) & $240.75(7.43)$ & Royal Gazette ${ }^{14}$ \\
\hline \multicolumn{3}{|l|}{ Costs of cardiovascular events } \\
\hline Fatal myocardial infarction & $256,875(7,93 I)$ & Anukoolsawat et al, $2006^{15}$ \\
\hline Nonfatal myocardial infarction & $176,842(5,460)$ & Anukoolsawat et al, $2006^{15}$ \\
\hline Stroke & $35,698(1,102)$ & Khiaocharoen et al, $2012^{16}$ \\
\hline
\end{tabular}

Abbreviations: mg, milligram; THB, Thai Baht; USD, United States Dollar (presented at year 2018).

$$
\text { Cost of control }=\frac{\text { cost of treatment }}{\begin{array}{l}
\text { percentage of patients achieving } \\
\text { the composite endpoint }
\end{array}}
$$

In part 1, the ratio of cost of control was calculated as the cost of control of liraglutide divided by the cost of control of sitagliptin. The ratio of cost of control $>1$ indicated the higher cost of liraglutide than sitagliptin treatment to achieve the composite endpoint.

The TECOS trial ${ }^{13}$ concluded that differences in adverse cardiovascular outcomes between the placebo and sitagliptin treatment were insignificant $(P>0.05)$ for intention-to-treat analysis. In contrast, liraglutide significantly reduced some adverse cardiovascular outcomes in the LEADER trial, ${ }^{11}$ including primary composite outcome [first occurrence of death from cardiovascular cause, non-fatal myocardial infarction, or non-fatal stroke (hazard ratio, HR; $95 \%$ confidence interval, $\mathrm{CI}=0.87$ (0.78-0.97)], death from cardiovascular causes [HR, $95 \%, \mathrm{CI}=0.78(0.66-0.93)]$ and myocardial infarction [(HR, 95\%, CI=0.86 (0.73-1.00)]. Consequently, the magnitude of reductions of incidence rate between the active treatment (either liraglutide or sitagliptin) and placebo groups were quantified. A positive number indicated reduced cardiovascular outcomes with the active treatment group compared with the placebo group. The incidence ratio was also calculated. An incidence ratio of $>1$ indicated a greater reduction in cardiovascular outcomes with liraglutide compared with sitagliptin. The mathematical equations for calculation were as follows:

Incidence rate reduction in liraglutide group=number of events in placebo group-number of events in liraglutide group

Incidence rate reduction in sitagliptin group=number of events in placebo group-number of events in sitagliptin group

$$
\text { Incidence ratio }=\frac{\begin{array}{l}
\text { incidence rate reduction in liraglutide } \\
\text { group }
\end{array}}{\begin{array}{l}
\text { incidence rate reduction in sitagliptin } \\
\text { group }
\end{array}}
$$

In addition to cost of control, the ratio of cost of control between cost of control for liraglutide and sitagliptin was calculated. The ratio of $>1$ indicated the higher cost of liraglutide than sitagliptin treatment. To derive the reduction in incidence rate in the liraglutide group, the difference between the number of events in the placebo group and number of events in the liraglutide group was calculated.

The monetary value of the benefits from reducing the incidence of composite endpoint (death from cardiovascular cause, non-fatal myocardial infarction, and non-fatal stroke) was also calculated by multiplying the proportion of such events, reported in the TECOS ${ }^{13}$ and LEADER trials, ${ }^{11}$ by their treatment costs. However, the cost of saving life was not included. The difference in total costs incurred between the two drug treatments were calculated as the cost savings.

\section{Results}

\section{Part I}

Treatment with liraglutide (1.2 and $1.8 \mathrm{mg}$ daily doses) costs 4.91- and 7.37-times more, respectively, than with sitagliptin $100 \mathrm{mg}$ (Table 3). However, the $1.8 \mathrm{mg}$ liraglutide treatment resulted in $49 \%$ of patients achieving the composite endpoint of T2DM control ( $\mathrm{HbAlc}<7.0 \%$, with no reported hypoglycemia and no body weight gain), the highest percentage among all the treatments. In this respect, liraglutide $1.2 \mathrm{mg}$ and $1.8 \mathrm{mg}$ daily doses were 2.09- and 2.63-times more effective, respectively, compared with sitagliptin $100 \mathrm{mg}$ (Table 1). Consequently, the cost per controlled patient using 
Table 3 Part I base case analysis

\begin{tabular}{|c|c|c|c|}
\hline & \multicolumn{3}{|l|}{ Treatment } \\
\hline & Liraglutide $1.2 \mathrm{mg}$ & Liraglutide $1.8 \mathrm{mg}$ & Sitagliptin $100 \mathrm{mg}$ \\
\hline Cost of treatment per patient (THB/USD per year) & $58,583(1,809)$ & $87,874(2,713)$ & $11,928(368)$ \\
\hline Ratio of treatment cost ${ }^{a}$ & 4.91 & 7.37 & \\
\hline Percentage of patients achieving the composite endpoint ${ }^{b}$ & 38.9 & 49.0 & 18.60 \\
\hline Ratio of composite endpoint ${ }^{c}$ & 2.09 & 2.63 & \\
\hline Cost per controlled patient $(\mathrm{THB})^{\mathrm{d}}$ & $150,598(4,650)$ & $179,334(5,537)$ & $64,130(1,980)$ \\
\hline \multicolumn{4}{|l|}{ (USD) } \\
\hline Ratio of cost of control ${ }^{e}$ & 2.35 & 2.80 & \\
\hline
\end{tabular}

Notes: ${ }^{a}$ Ratio of treatment cost $=$ cost of liraglutide treatment/cost of sitagliptin treatment. ${ }^{b}$ Composite endpoint defined as achieving $\mathrm{HbA} / \mathrm{c}<7.0 \%$, no hypoglycemia, and no weight gain. ${ }^{\mathrm{C}}$ Ratio of composite endpoint $=$ number of patients achieving the composite endpoint in liraglutide group/those in sitagliptin group. ${ }^{\mathrm{d}}$ Cost of control $=$ cost of treatment per patient/percentage of patients achieving the composite endpoint. ${ }^{e}$ Ratio of cost of control = cost of control in liraglutide group/cost of control in sitagliptin group. Abbreviations: mg, milligram; THB, Thai Baht; USD, United States Dollar (presented at year 2018).

liraglutide was only 2.35 - and 2.80-times higher (1.2 and $1.8 \mathrm{mg}$ daily doses, respectively), compared with sitagliptin $100 \mathrm{mg}$ (Table 3).

\section{Part II}

Liraglutide (1.8 $\mathrm{mg}$ daily dose) reduced the overall incidence of composite endpoint (cardiovascular death, nonfatal myocardial infarction, and non-fatal stroke) 12.50-times more effectively than sitagliptin $100 \mathrm{mg}$ did, by 0.50 (sitagliptin) vs 0.04 (liraglutide) events per 100 patients per year. This resulted in a lower cost per event reduction for liraglutide, compared with sitagliptin [175,748 THB (5,426 USD) vs 298,205 THB $(9,207$ USD), respectively] (Table 4). Sitagliptin, in effect, increased death from cardiovascular causes slightly, compared with the placebo (by 0.05 incidents per 100 patientsyear), whereas liraglutide reduced it by 0.40 incidents per 100 patients-year) $(P=0.007)$. Consequently, the incidence ratio could not be calculated. Liraglutide $1.8 \mathrm{mg}$ also reduced incidence of myocardial infarction 3.3-times more than sitagliptin did (compared with their respective placebos). However, incidence of myocardial infarction remained higher with liraglutide than with sitagliptin, although the statistical significance of the result was borderline $(P=0.046){ }^{11}$ Consequently, the cost per event-reduction of myocardial infarction was 2.21-times higher with liraglutide than with sitagliptin (Table 4).

When the economic costs of adverse cardiovascular outcomes that constituted the cardiovascular composite endpoint were taken into account, treatment with liraglutide $1.8 \mathrm{mg}$ reduced such costs by $32,512 \mathrm{THB}(1,004$ USD) per 100 patients per year compared with the placebo. This was 20,085 THB (620 USD) more than the cost savings from treatment with sitagliptin $100 \mathrm{mg}[12,427$ THB (384 USD)] (Table 5).

\section{Discussion}

The significant clinical benefits of liraglutide are substantiated by strong clinical evidence; ${ }^{11}$ however, the cost of the drug must also be considered, when comparing it with commonly used incretin-based oral anti-diabetic drugs, such as sitagliptin. Therefore, this study considered both the costs and benefits of the drug, compared with sitagliptin. Part 1 of the study revealed that, once the clinical benefits are included (achieving the composite endpoint: $\mathrm{HbA} 1 \mathrm{c}<7.0 \%$, no hypoglycemia and no weight gain), the costs of the liraglutide treatments (1.8 and $1.2 \mathrm{mg}$ daily) per controlled patient were 2.35- and 2.80-times higher, respectively, than for sitagliptin $100 \mathrm{mg}$. This result contrasts with a similar Canadian study ${ }^{18}$ that reported lower costs to achieve the same clinical endpoint of liraglutide (1.8 and $1.2 \mathrm{mg}$ daily) compared with sitagliptin (100 mg daily). This may have been due to a smaller difference in the annual medication costs between the two drugs in Canada (liraglutide $1.2 \mathrm{mg}$ is only 1.9-times more expensive than sitagliptin $100 \mathrm{mg}$, compared with 4.9- and 7.4-times more expensive [1.2 and $1.8 \mathrm{mg} /$ day, respectively) in Thailand]. Therefore, the clinical benefits provided by treatment with liraglutide treatment could outweigh its high medical cost in Canada.

When cardiovascular outcomes are included in the evaluation, ${ }^{11,13}$ liraglutide reduced the incidence rate of major adverse cardiovascular events (MACE) - cardiovascular death, non-fatal myocardial infarction, and non-fatal stroke - by 12.50-times compared to sitagliptin. Ultimately, this meant that for every 1 THB spent on sitagliptin, only 
Table 4 Part II base case analysis - cardiovascular outcomes

\begin{tabular}{|c|c|c|}
\hline \multirow[t]{2}{*}{ Variables } & \multicolumn{2}{|l|}{ Treatment } \\
\hline & Liraglutide $1.8 \mathrm{mg}$ & Sitagliptin 100 mg \\
\hline \multicolumn{3}{|l|}{ Cost } \\
\hline Cost of treatment/year THB (USD) & $87,874(2,7 \mid 3)$ & $11,928(368)$ \\
\hline Ratio of treatment cost ${ }^{\mathrm{a}}$ & \multicolumn{2}{|l|}{7.37} \\
\hline \multicolumn{3}{|l|}{ Composite endpoint ${ }^{b}$} \\
\hline Incidence rate reduction & 0.50 & 0.04 \\
\hline Incidence ratio ${ }^{d}$ & 12.50 & \\
\hline Cost of control (THB per event reduction) ${ }^{c}$ & $175,748(5,426)$ & $298,200(9,207)$ \\
\hline Ratio of cost of control ${ }^{\mathrm{e}}$ & \multicolumn{2}{|l|}{0.59} \\
\hline \multicolumn{3}{|l|}{ Death from cardiovascular causes } \\
\hline Incidence rate reduction & 0.4 & -0.05 \\
\hline Cost of control (THB per event reduction $)^{c}$ & $219,684(6,782)$ & $\mathrm{n} / \mathrm{a}$ \\
\hline Incidence ratio ${ }^{d}$ & $\mathrm{n} / \mathrm{a}$ & \\
\hline Ratio of cost of control ${ }^{e}$ & \multicolumn{2}{|l|}{$\mathrm{n} / \mathrm{a}$} \\
\hline \multicolumn{3}{|l|}{ Myocardial infarction } \\
\hline Incidence rate reduction & 0.30 & 0.09 \\
\hline Cost of control (THB per event reduction) ${ }^{c}$ & $292,913(9,043)$ & $132,536(4,092)$ \\
\hline Incidence ratio ${ }^{d}$ & 3.33 & \\
\hline Ratio of cost of control ${ }^{\mathrm{e}}$ & 2.21 & \\
\hline
\end{tabular}

Notes: ${ }^{a}$ Ratio of treatment cost $=$ cost of liraglutide treatment/cost of sitagliptin treatment. ${ }^{b}$ Composite endpoint defined as death from cardiovascular causes, nonfatal myocardial infarction, nonfatal stroke. ${ }^{c}$ Cost of control $=$ cost of each treatment/incidence rate reduction in each treatment. ${ }^{d}$ Incidence ratio $=$ incidence rate of liraglutide treatment/incidence rate of sitagliptin treatment. ${ }^{e}$ Ratio of cost of control $=$ cost of control for liraglutide treatment/cost of control for sitagliptin treatment. ${ }^{f}$ Patients in the sitagliptin group had a higher death rate from cardiovascular causes than those in the placebo group.

Abbreviations: mg, milligram; THB, Thai Baht; USD, United States Dollar (presented at year 2018).

Table 5 Economic benefit from the reduction of cardiovascular outcome

\begin{tabular}{|c|c|c|c|c|}
\hline \multirow[t]{2}{*}{ Variables } & \multicolumn{2}{|c|}{ LEADER trial ${ }^{\prime \prime}$} & \multicolumn{2}{|c|}{ TECOS trial ${ }^{13}$} \\
\hline & $\begin{array}{l}\text { Liraglutide } \\
1.8 \mathrm{mg}\end{array}$ & Placebo & $\begin{array}{l}\text { Sitagliptin } \\
100 \mathrm{mg}\end{array}$ & Placebo \\
\hline $\begin{array}{l}\text { Incidence rate of cardiovascular composite endpoint }{ }^{\mathrm{a}} \text { (no. of events per } 100 \\
\text { patients/year) }\end{array}$ & $3.4^{\mathrm{b}}$ & $3.9^{\mathrm{b}}$ & $3.58^{\mathrm{c}}$ & $3.62^{\mathrm{c}}$ \\
\hline Costs composite endpoint ${ }^{d}$ THB (USD) & $\begin{array}{l}302,296 \\
(9,333)\end{array}$ & $\begin{array}{l}334,808 \\
(10,337)\end{array}$ & $\begin{array}{l}267,407 \\
(8,256)\end{array}$ & $\begin{array}{l}279,834 \\
(8,640)\end{array}$ \\
\hline Cost savings from composite endpoint reduction ${ }^{\mathrm{e}}$ THB (USD) & \multicolumn{2}{|c|}{$32,512(1,004)$} & \multicolumn{2}{|l|}{ 12,427 (384) } \\
\hline Cost savings from liraglutide treatment ${ }^{f}$ THB (USD) & \multicolumn{4}{|l|}{$20,085(620)$} \\
\hline
\end{tabular}

Notes: ${ }^{a}$ Composite endpoint defined as death from cardiovascular causes, nonfatal myocardial infarction, or nonfatal stroke. ${ }^{\text {b}}$ The proportion of each cardiovascular outcome in the liraglutide treatment group was $29.8 \%$ for cardiovascular death, $45.2 \%$ for nonfatal myocardial infarction and $25.0 \%$ for nonfatal stroke while the proportion in the placebo group was $32.7 \%$ for cardiovascular death, $43.8 \%$ for nonfatal myocardial infarction, and $23.5 \%$ for nonfatal stroke. ${ }^{\circ}$ The proportion of each cardiovascular outcome in the sitagliptin treatment group was $42.0 \%$ for cardiovascular death, $38.3 \%$ for nonfatal myocardial infarction, and $19.7 \%$ for nonfatal stroke, while the proportion in the placebo group was $39.3 \%$ for cardiovascular death, $39.4 \%$ for nonfatal myocardial infarction, and $21.3 \%$ for nonfatal stroke. ${ }^{\mathrm{d}}$ Cost incurred from composite endpoint $=$ cost of each cardiovascular outcome*The proportion of that event that occurred. However, cost of cardiovascular death was not included due to limited data. ${ }^{\text {e }}$ Cost savings from composite endpoint reduction = cost incurred in the placebo group-cost incurred in the treatment group. ${ }^{\mathrm{f} C o s t}$ savings from liraglutide treatment $=$ cost-saving from liraglutide treatment-cost-saving from sitagliptin treatment.

Abbreviations: no, number; THB, Thai baht; USD, United States Dollar (presented at year 2018). 
0.59 THB needs to be spent on liraglutide, to bring about control of a patient in terms of reducing MACE. After converting the lowered incidence rate of cardiovascular composite endpoint outcomes into a monetary value, liraglutide yielded cost savings of 20,085 THB (620 USD) per 100 patients per year compared with sitagliptin.

In addition, liraglutide $(1.8 \mathrm{mg})$ reduces mortality due to cardiovascular causes amongst T2DM patients significantly $(P=0.007),{ }^{11}$ whereas sitagliptin $(100 \mathrm{mg})$ had no significant effect $(P=0.71) .{ }^{13}$ If reduction of cardiovascular death is taken into account, the cost of control with liraglutide is much lower than that with sitagliptin.

Although liraglutide reduced myocardial infarction more than sitagliptin did, the cost of control ratio was 2.21, indicating that the cost of using liraglutide was more than double that of sitagliptin per event reduction of myocardial infarction.

Several limitations with this study should be discussed, the most notable of which is the absence of a head-to-head clinical trial comparing cardiovascular outcomes between liraglutide and sitagliptin. Although a meta-analysis has been published recently, ${ }^{19}$ comparing the effects of sodium-glucose cotransporter 2 inhibitors, GLP-1 receptor agonists, and dipeptidyl peptidase 4 (DPP-4) inhibitors on all causes of mortality among T2DM patients, we decided not to use its findings, in terms of cardiovascular outcomes, due to the following reasons. Firstly, most studies included in the meta-analysis did not aim to measure cardiovascular outcomes as the primary endpoint. Of the 14 studies included in the meta-analysis that compared between DPP-4 inhibitors and GLP-1 agonists, fewer than half of them compared between liraglutide and sitagliptin. Therefore, the cardiovascular outcomes of liraglutide and sitagliptin were based on the landmark clinical trials, LEADER ${ }^{11}$ and TECOS. ${ }^{13}$ Both of these trials enrolled T2DM patients at high risk of cardiovascular disease, even though patients who received the placebo in the LEADER trial ${ }^{11}$ were more likely to have cardiovascular events than those in the TECOS trial were. ${ }^{13}$ To address this issue, cardiovascular outcomes that occurred when using the two drugs were compared with their respective placebos, and not with each other. Secondly, although death from cardiovascular causes was included among the outcomes that constituted the composite endpoint, there is no data on the monetary value of preventing cardiovascular death in Thailand. Consequently, monetary benefit was calculated from the reduction in non-fatal myocardial infarction and non-fatal stoke only.
Furthermore, in the LEADER trial, ${ }^{20}$ liraglutide significantly reduced composite renal outcomes (including death due to renal cause, renal replacement therapy, doubling of serum creatinine, and new onset of macroalbuminuria) $(P=0.003)$; whereas, in the TECOS trial, ${ }^{13}$ sitagliptin had no significant effect. When such savings are added to those found by the present study (based on cardiovascular outcomes), the total cost savings would be considerably increased.

Although treatment with liraglutide is expensive relative to other oral anti-diabetic drugs and reimbursement is limited in many healthcare systems, including Thailand, its use when other treatments fail or lead to significant side-effects may be suitable. Other studies $^{21,22}$ have shown that liraglutide reverses pronounced insulin-associated weight gain, decreases insulin requirements, and improves glycemic control in T2DM patients. Such benefits may also result in additional cost savings, which are not included in the present study.

\section{Conclusion}

This study has identified the clinical benefits of liraglutide, namely, HbA1c $<7.0 \%$, no hypoglycemia, and no weight increase, partially offset in its high price. In addition, when cardiovascular benefits are taken into account in monetary terms, liraglutide yields cost savings. Liraglutide should, therefore, be considered as an appropriate alternative to sitagliptin, particularly for T2DM patients with high cardiovascular risks.

\section{Acknowledgments}

The authors thank Novo Nordisk Pharma (Thailand) Limited for funding this research. The sponsor assisted in literature review and organized the meetings, but played no part in directing the study design, selecting input parameters, analyzing data, or preparing the manuscript.

\section{Disclosure}

$\mathrm{CD}, \mathrm{NK}$, and UP declare no conflicts of interest in this work. AG is an employee of Novo Nordisk Pharma (Thailand) Ltd. PC is a previous employee of Novo Nordisk Pharma (Thailand) Ltd. The authors report no other conflicts of interest in this work. 


\section{References}

1. Hu FB. Globalization of diabetes: the role of diet, lifestyle, and genes. Diabetes Care. 2011;34(6):1249-1257. doi:10.2337/dc11-0442

2. Papier K, Jordan S, D`Este C, et al. Incidence and risk factors for type 2 diabetes mellitus in transitional Thailand: results from the Thai cohort study. BMJ Open. 2016;6(12):e014102. doi:10.1136/bmjopen2016-014102

3. Aekplakorn W, Puckcharern H, Thaikla K, Satheannoppakao W. Thai National Health Examination Survey V. Bangkok: Health Systems Research Institute; 2014.

4. Strategy and Planning Division, Ministry of Public Health. Public Health Statistics. A.D.2016. Bangkok: Ministry of Public Health; 2017.

5. Deerochanawong C, Ferrario A. Diabetes management in Thailand: a literature review of the burden, costs, and outcomes. Global Health. 2013;9:11. doi:10.1186/1744-8603-9-11

6. Brouwer WB, Rutten FF, Koopmanschap MA. Costing in economic evaluations. In: Drummond M, McGuire A, editors. Economic Evaluation in Health Care: Merging Theory with Practice. New York: Oxford University Press; 2001:68-93.

7. Chatterjee S, Riewpaiboon A, Piyauthakit P, et al. Cost of diabetes and its complications in Thailand: a complete picture of economic burden. Health Soc Care Community. 2011;19(3):289-298. doi:10.1111/j.1365-2524.2010.00981.x

8. Pratley RE. Overview of glucagon-like peptide-1 analogs and dipeptidyl peptidase-4 inhibitors for type 2 diabetes. Medscape J Med. 2008;10(7):171.

9. Pratley RE, Nauck M, Bailey T, et al. Liraglutide versus sitagliptin for patients with type 2 diabetes who did not have adequate glycaemic control with metformin: a 26-week, randomised, parallel-group, open-label trial. Lancet (London, England). 2010;375(9724):1447-1456. doi:10.1016/ S0140-6736(10)60307-8

10. Pratley R, Nauck M, Bailey T, et al. One year of liraglutide treatment offers sustained and more effective glycaemic control and weight reduction compared with sitagliptin, both in combination with metformin, in patients with type 2 diabetes: a randomised, parallel-group, open-label trial. Int J Clin Pract. 2011;65(4):397-407. doi:10.1111/ j.1742-1241.2011.02656.x

11. Marso SP, Daniels GH, Brown-Frandsen K, et al. Liraglutide and cardiovascular outcomes in type 2 diabetes. N Engl J Med. 2016;375 (4):311-322. doi:10.1056/NEJMoa1603827
12. Singh AK, Singh R. Recent cardiovascular outcome trials of antidiabetic drugs: a comparative analysis. Indian J Endocrinol Metab. 2017;21(1):4-10. doi:10.4103/2230-8210.196026

13. Green JB, Bethel MA, Armstrong PW, et al. Effect of sitagliptin on cardiovascular outcomes in type 2 diabetes. $N$ Engl J Med. 2015;373 (3):232-242. doi:10.1056/NEJMoa1501352

14. National Drug System Development Committee. Median Drug Price Annoucement. Bangkok: Office of the Prime Minister Bangkok: Royal Gazette in Thailand; 2018.

15. Anukoolsawat P, Sritara P, Teerawattananon Y. Costs of lifetime treatment of acure coronary syndrome at Ramathibodi hospital. Thai Heart J. 2006;19:132-143.

16. Khiaocharoen O, Pannarunothai S, Zungsontiporn C. Cost of acute and sub-acute care for stroke patients. J Med Assoc Thai. 2012;95 (10):1266-1277.

17. Bank of Thailand. Foreign Exchange Rate 2018. Available from: https://www.bot.or.th/English/FinancialMarkets/layouts/Application/ ExchangeRate/ExchangeRate.aspx. Accessed September 24, 2018.

18. Skovgaard R, Jon Ploug U, Hunt B, Valentine WJ. Evaluating the cost of bringing people with type 2 diabetes mellitus to multiple targets of treatment in Canada. Clin Ther. 2015;37(8):1677-1688. doi:10.1016/j.clinthera.2015.05.496

19. Zheng SL, Roddick AJ, Aghar-Jaffar R, et al. Association between use of sodium-glucose cotransporter 2 inhibitors, glucagon-like peptide 1 agonists, and dipeptidyl peptidase 4 inhibitors with all-cause mortality in patients with type 2 diabetes: a systematic review and meta-analysis. Jama. 2018;319(15):1580-1591. doi:10.1001/ jama.2018.3024

20. Mann JFE, Orsted DD, Brown-Frandsen K, et al. Liraglutide and renal outcomes in type 2 diabetes. $N$ Engl J Med. 2017;377 (9):839-848. doi:10.1056/NEJMoa1616011

21. de Wit HM, Vervoort GM, Jansen HJ, de Grauw WJ, de Galan BE, Tack CJ. Liraglutide reverses pronounced insulin-associated weight gain, improves glycaemic control and decreases insulin dose in patients with type 2 diabetes: a 26 week, randomised clinical trial (ELEGANT). Diabetologia. 2014;57(9):1812-1819. doi:10.1007/ s00125-014-3302-0

22. de Wit HM, Vervoort GM, Jansen HJ, de Galan BE, Tack CJ. Durable efficacy of liraglutide in patients with type 2 diabetes and pronounced insulin-associated weight gain: 52-week results from the effect of liraglutide on insulin-associated wEight GAiN in patients with type 2 diabetes' (ELEGANT)randomized controlled trial. J Intern Med. 2016;279(3):283-292. doi:10.1111/joim.12447

\section{Publish your work in this journal}

ClinicoEconomics and Outcomes Research is an international, peerreviewed open-access journal focusing on Health Technology Assessment, Pharmacoeconomics and Outcomes Research in the areas of diagnosis, medical devices, and clinical, surgical and pharmacological intervention. The economic impact of health policy and health systems organization also constitute important areas of coverage. The manuscript management system is completely online and includes a very quick and fair peer-review system, which is all easy to use. Visit http://www.dovepress.com/testimonials.php to read real quotes from published authors. 